Cite this paper: KL Pitts, S Abu-Mallouh, M Fenech, journal of the mechanical behavior of biomedical materials 17, 333-336 https://doi.org/10.1016/j.jmbbm.2012.07.007

\title{
Contact Angle Study of Blood Dilutions on Common Microchip Materials
}

\author{
K. L. Pitts, ${ }^{a}$ S. Abu-Mallouh, ${ }^{\mathrm{b}}$ M. Fenech ${ }^{\mathrm{a}, \mathrm{b}, *}$
}

${ }^{a}$ Department of Chemical and Biological Engineering, University of Ottawa, 161 Louis-Pasteur, Ottawa, K1N 6N5, Canada

${ }^{b}$ Department of Mechanical Engineering, University of Ottawa, 161 Louis-Pasteur, Ottawa, K1N 6N5, Canada *corresponding author: mfenech@uOttawa.ca, Tel: 1- 613-562-5800 (x1924), Fax: 1-613-5625177

\begin{abstract}
Biocompatible polymers are commonly used to fabricate microfluidic channels for the study of biological flows such as blood microflows. The most common of these materials is polydimethylsiloxane (PDMS) which is very hydrophobic. Oxygenated plasma is advocated to treat the PDMS with reported decreases in contact angle i.e increase the hydrophilicity of the material in order to make the liquid to flow easily. All contact angle studies have been reported with water. Here the contact angles of blood suspensions, in saline and native plasma, are compared to each other and water on common microfluidic chip materials. The hydrophilic effect of plasma-treatment on PDMS is not found to be as significant with blood suspensions as it is with
\end{abstract}


water. Red blood cells suspended in native plasma are found to have a greater contact angle than those suspended in saline

\section{Introduction}

Microfluidic chips are frequently fabricated in order to study biological flows such as blood. These microchips are scaled to mimic the microcirculation in the body, where vessels are on the order of $100 \mu \mathrm{m}$ in diameter [1], or for blood tests in lab-on-a-chip devices like immunoassays. For the lab-on-a-chip devices the hydrophilicity or "wettability" of the material is of great importance as the blood products are usually driven by capillary effect. Regarding the in vitro study of the microcirculation, the hydrophilic behavior of the material will modify important physiological parameters such as the shear rate at the wall. The surface interactions required to generate blood oxygenation in a microchip environment also depend on the "wettability" of surface [2]. Contact angle is used here as a measure of "wettability". The blood products used in these studies could be plasma, whole blood, suspensions of red blood cells (RBCs) in native plasma, or in artificial plasma such as saline. These suspensions avoid the clotting that occurs with platelets and whole blood. Glass is generally avoided as a medium for these chips due to its tendency to cause echinocytosis in red blood cells (RBCs). Commonly used biocompatible materials are polydimethylsiloxane (PDMS) and poly(methyl methacrylate)(PMMA).For biological applications of PDMS microchips, fabrication is generally done through soft lithography $[3,4,5]$. Bélanger and Marois reviewed the research into the biocompatibility and hemocompatibility of PDMS as a biomaterial. Their review indicates that PDMS has good hemocompatibility and other research indicates porcine blood samples are a good indicator of human blood, at least in the case of platelet interaction [6].In addition to biocompatibility tests, there has been significant research 
in the surface modification of PDMS such as oxygen plasma treatment, to change the inherent hydrophobic nature of the polymer [7]. While not used as widely PMMA has been put forward as an alternative to PDMS as a channel material, as reviewed by Chen, Zhang and Chen in 2008, in part because it was thought to be the most hydrophilic of the biocompatible polymer options [8].Another thermoplastic previously suggested for use in blood research was polypropylene (PP) [9]. The problems associated with using thermoplastics like PMMA or PP is the high processing temperatures and difficulty creating inexpensive, small-volume runs of a microchip, which is why PDMS is so widely used in research.

When fabricating channels out of PDMS, oxygen plasma treatment is as a way to alter its naturally hydrophobic surface properties to a more hydrophilic surface. This is supported by contact angle studies done by Bhattacharya et al.[10].While oxygen plasma treatment is documented to work for water or water-based suspensions; the increase in hydrophilicity has not been demonstrated for blood solutions. This paper aims to test the effect of various common channel materials on the contact angle with changing the blood product's composition and to tabulate this data to be used as a reference for the microfluidics community.

\section{Materials and methods}

For this study six samples of fresh, healthy porcine blood were obtained from an accredited slaughterhouse. All samples were centrifuged for ten minutes, three times at 3000 RPM. After each round of centrifugation all the plasma was separated from the sample, and held (each sample's plasma was held individually).After the removal of the plasma, all white blood cells, and platelets were removed leaving only the RBCs. For the second and third round of centrifugation, some native plasma was returned to the samples, and they were gently mixed before centrifugation. After 
the third round, RBCs of each sample were obtained. These RBC were suspended in either PBS or their own native plasma at hematocrits $(\mathrm{H})$ of $10 \%, 20 \%$ and $30 \%$.Additional plasma from each sample was held for separate testing. Surfaces tested were glass (clean glass microscope slides), PDMS in a 10:1 ratio of polymer to curing agent (Sylgard-184, Dow, USA), PDMS in a 10:1 ratio exposed to oxygen plasma for 45 seconds (Anatech, LTD, USA) and clean PMMA. The oxygenplasma treated PDMS portions were cut from the same sample as the untreated PDMS.

All of the testing was done using the VCA-Optima (AST Products, Inc, USA) contact angle set-up. For each measurement at least three different samples were tested, and each test was repeated 3 times. Thus the contact angles reported are the average of the left and right contact

angle for 9 different images, resulting in 18 measurements. Exceptions are water, which was 12 measurements each surface; PBS 6 measurements each surface; $H=20$ in PBS 12 measurements on PMMA, $\mathrm{H}=30$ in plasma 14 measurements on plasma-treated PDMS, native plasma 12 measurements on PDMS and 14 on plasma-treated PDMS.

\section{Results and discussion}

Results of the contact angle studied are summarized in Table 1.A direct comparison on the base fluids (native plasma or PBS) with distilled water is shown in Figure 1.Error bars in the figure are the standard deviations listed in Table 1.Figures 2 and 3 highlight the results of increasing the hematocrit on the contact angle of the blood on the various surfaces.

Using artificial suspending fluids like PBS removes the native proteins and associated molecules in the plasma. Biological samples inherently have greater variance than artificial 
solutions created in a controlled environment as protein content can vary from sample to sample within the same species. RBCs suspended in PBS solution therefore have less variation between samples since the suspending solution is identical for all the samples. This clearly has an effect on the contact angle of the fluid on certain surfaces, especially the plasma treated PDMS. It is especially interesting the see the increase in contact angle of the native plasma on its own, which is $109^{\circ}$ on plasma treated PDMS, while the water usually used to report the success of the plasma treatment has a contact angle of only $49^{\circ}$. There is almost no impact on the contact angle of the native plasma by the oxygen plasma treatment. The difference in contact angle fails a two-tailed $t$-test for unequal means in all concentrations of RBC at a confidence level of 99\%.With the actual blood suspensions shown in Figures 2 and 3, the PBS suspensions have a more pronounced reduction in contact angle with the oxygen plasma treatment than those suspended in native plasma $(P \leq 0.01)$ for all concentrations of RBC. PMMA seems to have a great advantage in Figures 1 and 3, as the contact angle is lower than for PDMS even when treated with plasma. This could be a viable option for hydrophilic microchips using blood suspensions in native plasma. In comparing the plasma treated PDMS with PMMA, at low hematocrit the difference between the contact angles on the two surfaces is not significant when suspended in saline, while it is significant when hematocrit reaches $30 \%(P \leq 0.01)$. For RBCs suspended in plasma, the difference in contact angles between plasma treated PDMS and PMMA is not statistically significant at $\mathrm{H}=10$, but it is for plasma alone and for $\mathrm{H}=20$ and $30(P \leq 0.01)$. Future research of blood flow in biocompatible microchips needs to consider the impact of protein or molecular interactions with their surfaces since the surface-fluid interaction could affect the final flow.

\section{Conclusions}


In this work we have tested the contact angle of suspensions of RBCs in saline versus native plasma on common microchip fabrication materials. It was demonstrated that the expectedincrease in hydrophobicity (measured by decreased contact angle) of PDMS treated with oxygen-plasma is not present when using blood. It was also demonstrated that the contact angle of the plasma and saline alone are significantly different on oxygen-plasma treated PDMS. Finally, it was shown that the contact angle of suspensions in RBCs in plasma have a higher contact angle on the treated PDMS than suspensions of RBCs in PBS.

\section{Acknowledgments}

The authors would like to thank Tom Henderson's Custom Meats of Chesterville, Ontario for the donation of the blood samples, Louis Tremblay for the technical support and The Natural Sciences and Engineering Research Council of Canada (NSERC) for funding the research.

\section{REFERENCES}

[1] Popel AS, Johnson PC. Microcirculation and hemorheology. Annu Rev Fluid Mech 2005 01/01;37(1):43-69.

[2] Atencia J, Beebe DJ. Controlled microfluidic interfaces. Nature 2005;437(7059):648-655.

[3] Cheung YK, Shiovitz D, Sia SK. Microfluidic-based lithography for fabrication of multicomponent biocompatible microstructures. In: Herold KE, Rasooly A, editors. Norfolk, UK: Caister Academic Press; 2009. p. 115.

[4] Sia SK, Whitesides GM. Microfluidic devices fabricated in poly(dimethylsiloxane) for biological studies. Electrophoresis 2003;24(21):3563-3576.

[5] McDonald JC, Whitesides GM. Poly(dimethylsiloxane) as a material for fabricating microfluidic devices. Acc Chem Res 2002;35(7):491-499.

[6] Bélanger M-, Marois Y. Hemocompatibility, biocompatibility, inflammatory and in vivo studies of primary reference materials low-density polyethylene and polydimethylsiloxane: A review. J Biomed Mater Res 2001;58(5):467-477. 
[7] Zhou J, Ellis AV, Voelcker NH. Recent developments in PDMS surface modification for microfluidic devices. Electrophoresis 2010;31(1):2-16.

[8] Chen Y, Zhang L, Chen G. Fabrication, modification, and application of poly(methyl methacrylate) microfluidic chips. Electrophoresis 2008;29(9):1801-1814.

[9] Kawamoto N, Terano M, Yui N. Blood-contacting properties of polypropylene surfaces. J Artif Organs 1998;1(1):4-9.

[10] Bhattacharya S, Datta A, Berg JM, Gangopadhyay S. Studies on surface wettability of poly(dimethyl) siloxane (PDMS) and glass under oxygen-plasma treatment and correlation with bond strength. J Microelectromech Syst 2005;14(3):590-597. 


\section{FIGURES AND TABLES}

Table 1.Contact angle data collected

\begin{tabular}{|c|c|c|c|c|c|c|c|c|}
\hline & \multicolumn{2}{|l|}{ glass } & \multicolumn{2}{|l|}{ PDMS } & \multicolumn{2}{|c|}{ p-PDMS } & \multicolumn{2}{|c|}{ PMMA } \\
\hline & avg & stdev & avg & stdev & avg & stdev & avg & stdev \\
\hline water & 9.1 & 3.8 & 111.1 & 2.9 & 31.1 & 4.9 & 91.3 & 2.6 \\
\hline PBS & 49.8 & 0.6 & 119.7 & 1.5 & 80.7 & 1.7 & 81.8 & 0.8 \\
\hline plasma & 27.0 & 6.9 & 113.7 & 6.5 & 109.6 & 8.6 & 72.1 & 1.9 \\
\hline H10, PBS & 32.9 & 16.5 & 108.2 & 8.2 & 85.6 & 14.0 & 80.4 & 8.4 \\
\hline $\mathrm{H} 10$, plasma & 41.7 & 10.1 & 80.3 & 35.9 & 88.1 & 26.7 & 72.6 & 7.8 \\
\hline $\mathrm{H} 20$, PBS & 23.6 & 5.7 & 99.6 & 8.5 & 71.6 & 23.7 & 80.1 & 7.7 \\
\hline $\mathrm{H} 20$, plasma & 41.0 & 7.7 & 98.0 & 19.0 & 102.4 & 10.1 & 67.5 & 8.8 \\
\hline H30, PBS & 32.5 & 8.4 & 105.9 & 11.3 & 74.9 & 25.3 & 74.5 & 6.0 \\
\hline $\mathrm{H} 30$, plasma & 35.6 & 6.0 & 91.6 & 27.2 & 92.8 & 22.6 & 60.4 & 17.2 \\
\hline
\end{tabular}

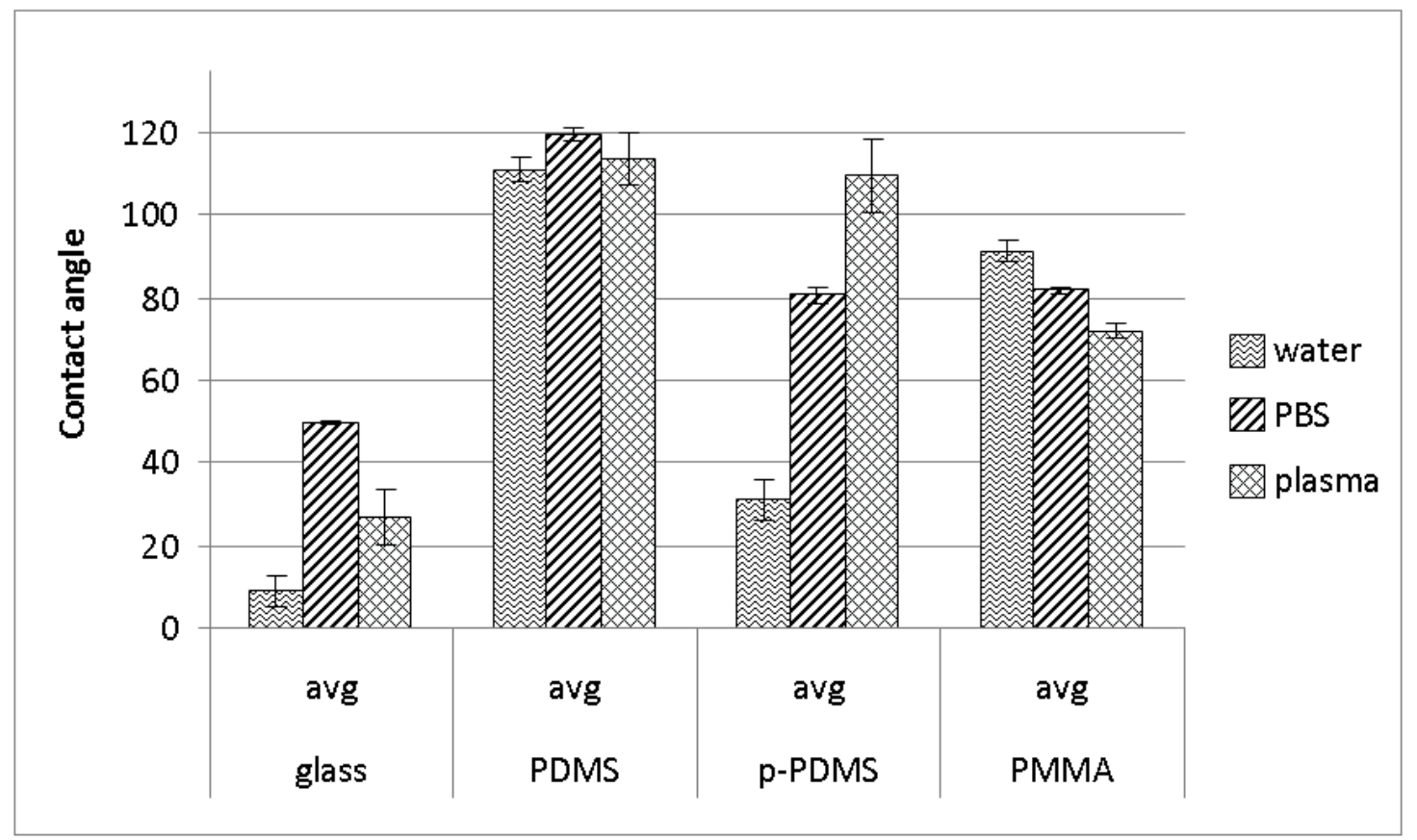

Figure 1. Contact angles of suspending fluids for the blood solutions, as compared to distilled water, on various microchip substrate materials 


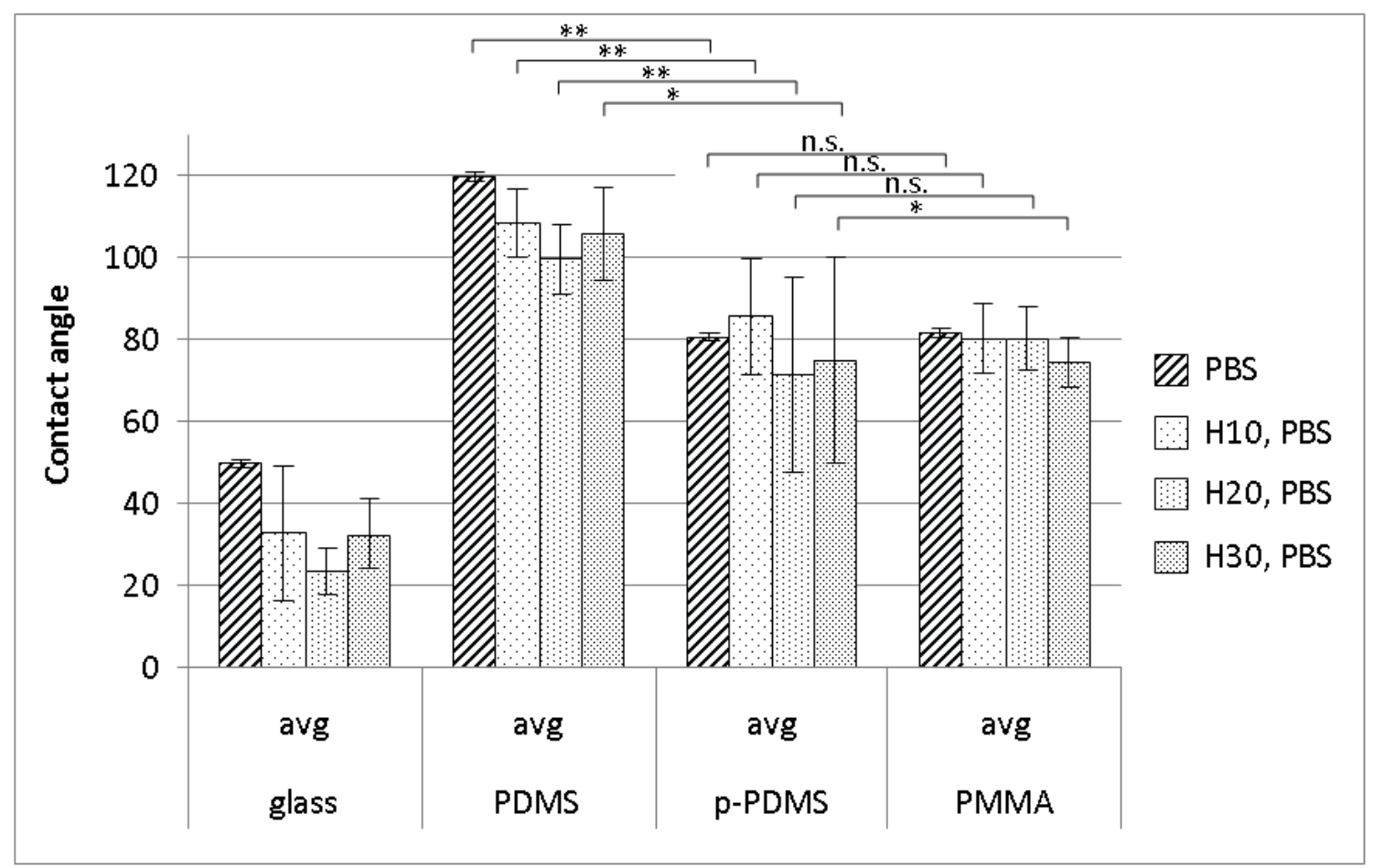

Figure 2. Contact angles of blood dilutions suspended in PBS, at hematocrit 10\%, 20\% and 30\%, on various microchip substrate materials, ${ }^{*} \mathrm{P} \leq 0.01, * * \mathrm{P} \leq 0.001$, n.s.: not significant. 


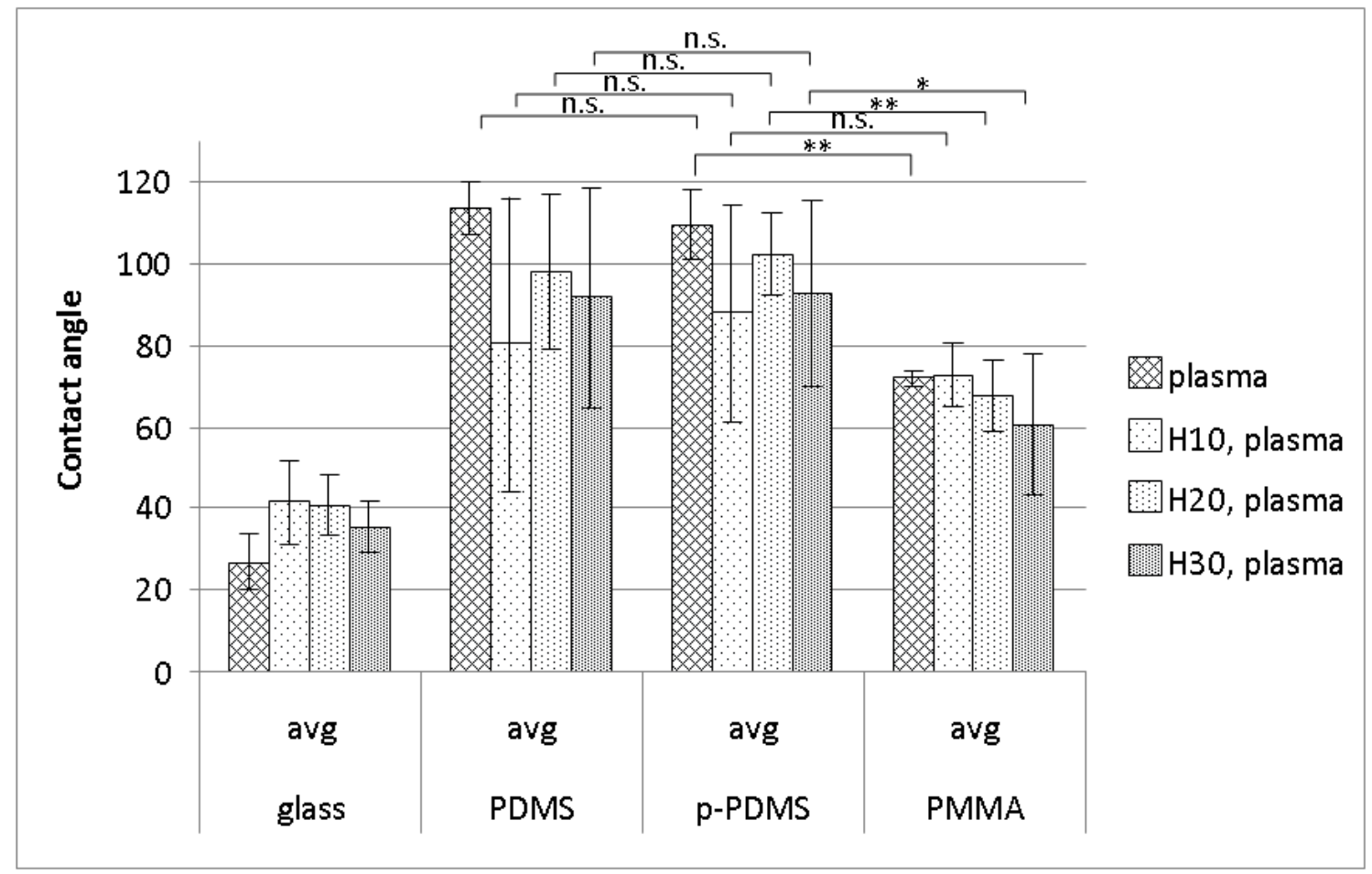

Figure 3. Contact angles of blood dilutions suspended in native plasma, at hematocrit 10\%, 20\% and $30 \%$, on various microchip substrate materials, $* \mathrm{P} \leq 0.01, * * \mathrm{P} \leq 0.001$, n.s.: not significant. 